\title{
Linac alignment algorithm: analysis on 1-to-1 steering
}

\author{
Yipeng Sun* and Chris Adolphsen \\ SLAC, Stanford, CA 94025, USA
}

\begin{abstract}
In a linear accelerator, it is important to achieve a good alignment between all of its components (such as quadrupoles, $\mathrm{RF}$ cavities, beam position monitors et al.), in order to better preserve the beam quality during acceleration. After the survey of the main linac components, there are several beam-based alignment (BBA) techniques to be applied, to further optimize the beam trajectory and calculate the corresponding steering magnets strength. Among these techniques the most simple and straightforward one is the one-to-one (1-to-1) steering technique, which steers the beam from quad center to center, and removes the betatron oscillation from quad focusing. For a future linear collider such as the International Linear Collider (ILC), the initial beam emittance is very small in the vertical plane (flat beam with $\gamma \epsilon_{y}=20-40 \mathrm{~nm}$ ), which means the alignment requirement is very tight. In this note, we evaluate the emittance growth with one-to-one correction algorithm employed, both analytically and numerically. Then the ILC main linac accelerator is taken as an example to compare the vertical emittance growth after 1-to-1 steering, both from analytical formulae and multi-particle tracking simulation. It is demonstrated that the estimated emittance growth from the derived formulae agrees well with the results from numerical simulation, with and without acceleration, respectively.
\end{abstract}

\section{Linac alignment problem}

The basic problem of linac misalignment can be sketched in Figure 1. The most ideal case is that all the elements of a linac are aligned on a straight line. In that case, there is no dispersive or wake-field-related emittance growth, if the beam is also injected on the axis. In practice, however, the linac components are scattered randomly with respect to the survey axis (treated as a straight line here) as shown in Figure 1. The beam will get a dipole kick when it pass by a quadrupole off center. If the offset is $\Delta y$, the kick is

$$
\Delta y^{\prime}=\frac{K_{1} \Delta y}{1+\delta_{p}}
$$

where $K_{1}$ denotes the normalized quadrupole strength, $K_{1}=\frac{d B_{y} / d y}{B \rho}, B \rho$ the beam rigidity which is proportional to beam energy, $\delta_{p}$ the energy offset of the particle.

In formula (1) it is shown that particle with different energy offset $\delta_{p}$ (and same initial coordinates) will get different dipole kick from the same quadrupole misalignment, and then perform different motion and have different trajectory. That will introduce dispersion and the dispersive emittance growth which is $\delta_{p}$-correlated. In comparison, without the quadrupole misalignments (dipole kicks), the chromatic difference only from the quadrupole kick is relatively small, and all the particles will have "same" betatron linear oscillation under the quadrupole focusing.

On the other hand, the beam centroid will follow the straight line without misalignment. With misalignment, it is also kicked and will perform "betatron" oscillation along the linac. Such an off-center distribution of the charges will generate wake field when it couples with the linac component impedance. The wake field has a longitudinal position correlation in the bunch, and will also introduce emittance growth. In this paper we will focus on the dispersive and

*Electronic address: yisun@slac.stanford.edu 


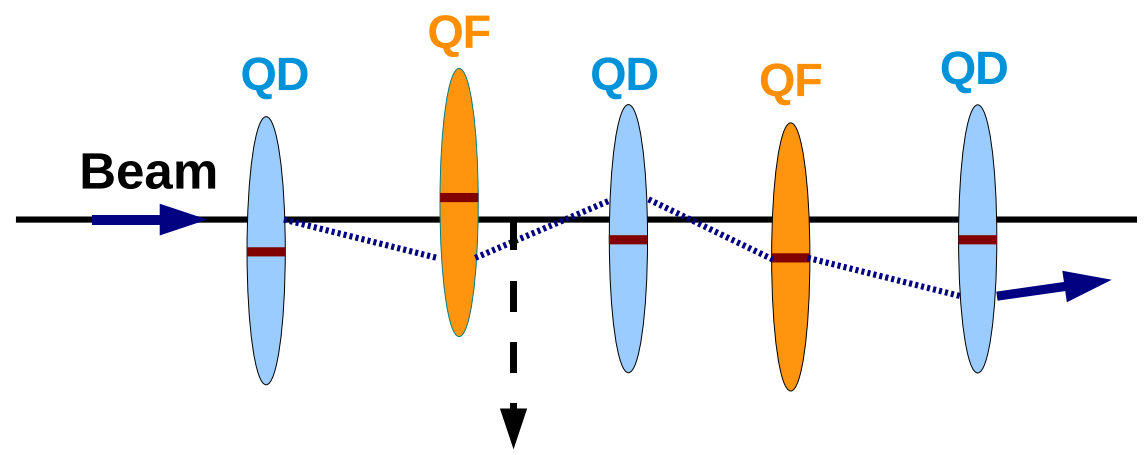

Alignment Axis

Figure 1: Sketch of linac misalignment problem.

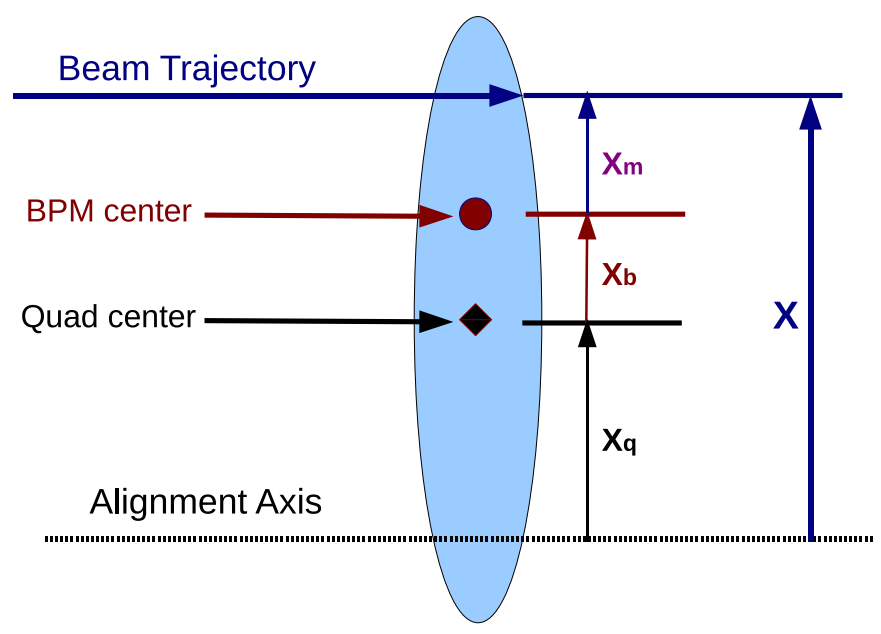

Figure 2: Different error sources for the linac beam-based alignment.

chromatic emittance growth studies.

Except the quadrupole misalignment, there are other error sources to be corrected in the beam-based alignment. In the linac, usually there is a Beam Position Monitor (BPM) and a pair of orbit corrector attached to each quadrupole magnet. The electrical BPM center may also have an offset with respect to the magnetic quadrupole center. BPM is used to measure the beam positions at the quadrupole locations, and it will also have a measurement error (BPM precision). In Figure 2, the quadrupole offset $x_{q}$, the BPM offset $x_{b}$ and the BPM measurement error $x_{m}$ are sketched together. The BPM reading is written as [1]

$$
X_{\text {read }}=X-X_{q}+X_{b}+X_{m}
$$




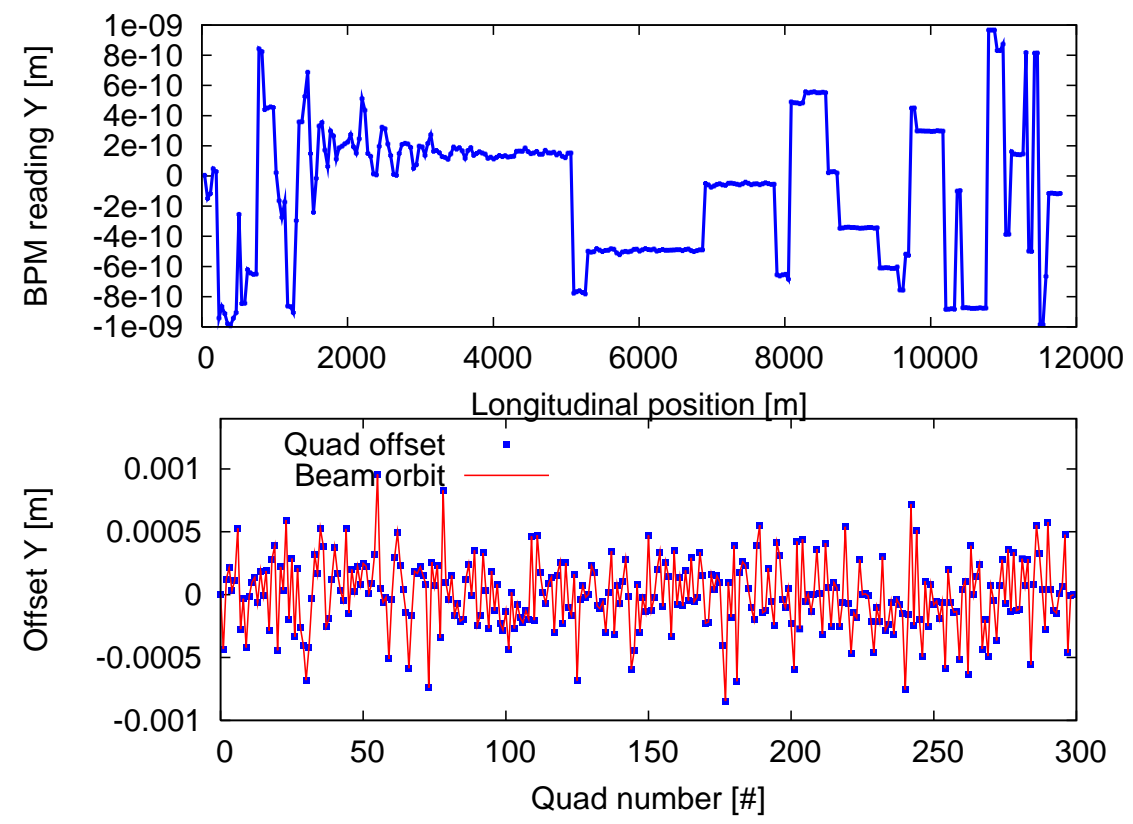

Figure 3: One to one correction, top: BPM readings; bottom: vertical quadrupole offsets and beam orbits.

\section{One to one correction scheme}

There are "global" and "local" emittance correction techniques. "Global" technique adds additional emittance dilution sources upstream of the measurement point, and let these effects cancel each other. The most straightforward one is to use emittance-bumps. "Local" correction technique measures and corrects the emittance dilution source locally.

There are several "local" linac misalignment measurement and correction algorithms. For example, Dispersion Free Steering (DFS) or Wake Field Free Steering (WFS) [2] measure the difference of two beam trajectories by varying some parameters (beam energy, quadrupole power supply et al.), and minimize this difference by using the combination of the orbit corrector family. Ballistic method [3] divides the linac into short bins, then align the BPM first and quadrupole afterwards, by using a ballistic beam. One to one correction is also used in the second step of this method.

In this paper we will focus on one to one correction. This technique uses the corrector pair to zero the next BPM readings downstream. If the BPM-to-quad offsets and BPM measurement error are not taken into account, the beam centroid will be kicked from one quadrupole center to another quadrupole center. The "betatron" oscillation of the beam centroid is effectively removed. In Figure 3, the application of one to one correction is shown for the vertical plane, where the BPM readings are almost zero, and the beam orbits equal the quadrupole offsets at the quadrupole center.

\section{Simulation}

\subsection{Code}

A new code is written in Fortran90. The basic function of this code is to define a lattice (or read in a lattice definition, from MADX), generate a bunch of (or many bunches of) macro-particles, and perform the tracking simulations. It can be used for either linear accelerator or storage rings. It can perform single-bunch or multi-bunch tracking, calculate the RMS emittance and other beam parameters at specified locations. The misalignment of the accelerator structures can be read in, or randomly generated with the RMS value defined. 
Up to now there are two BBA algorithms written in this code. One is so-called "global" correction algorithm which measures only one beam trajectory (in comparison, DFS uses two), and uses the modified transport matrix $R$ to establish a correlation between the quadrupoles and remove the emittance dilution. This algorithm is still being developed. Another one is the basic one to one correction algorithm.

Other correction algorithms, such as Dispersion Free Steering, Kick Minimization and Ballistic method will be added in the future.

The benchmark of the code is presented in Appendix B.

\subsection{Definition of dispersion and emittance}

The dispersion, projected emittance and linear dispersion corrected emittance can be calculated from the statistical property of the bunch, by using the macro-particle's 6-D coordinates, as shown in Appendix A [4].

\subsection{Generation of the bunch}

For single-bunch mode, a bunch of particles are generated randomly in six dimentional Gaussian distribution, namely $\left(x, x^{\prime}, y, y^{\prime}, c t, \delta_{p}\right)$. The number of macro-particles in a bunch is tested from 1000 to 1 million, and it is found that the simulation result has enough precision with 1000 particles per bunch. The transverse halos and longitudinal tails of the generated bunch are cut at $4 \sigma$.

For multi-bunch mode, the random number generator will be updated bunch by bunch.

\section{Analytical approach}

\subsection{Method One}

We start from the definition of the projected emittance:

$$
\gamma \epsilon_{y}=\gamma \sqrt{\left(<y^{2}>-<y>^{2}\right) \cdot\left(<y^{\prime 2}>-<y^{\prime}>^{2}\right)-\left(<y y^{\prime}>-<y><y^{\prime}>\right)^{2}}
$$

where $\gamma \epsilon_{y}$ denotes the normalized vertical emittance, $\gamma$ the relativistic factor, $y$ the vertical coordinate of one particle, $y^{\prime}$ the divergence (angle). The bracket means an average over all the macro-particles.

Let us consider the motion of the particles with respect to the centroid trajectory, we have

$$
\begin{aligned}
& <y>=0 \\
& <y^{\prime}>=0
\end{aligned}
$$

Further more, we choose two particles which have same transverse coordinates, but different energy offset. As the longitudinal coordinate with respect to the bunch center is almost "frozen" in a linac, we do not need to take it into account. The 6 -D coordinates of the two particles are $\left(x_{00}, x_{00}^{\prime}, y_{00}, y_{00}^{\prime}, c t, 0\right)$, and $\left(x_{00}, x_{00}^{\prime}, y_{00}, y_{00}^{\prime}, c t, \delta_{p}\right)$. Assume after passing half cell (one quad) in the linac, the difference of the transverse coordinates between these two particles are $\left(\Delta x, \Delta x^{\prime}, \Delta y, \Delta y^{\prime}\right)$, and the coordinates of particle 1 are $\left(x_{0}, x_{0}^{\prime}, y_{0}, y_{0}^{\prime}, c t, 0\right)$, the coordinates of particle 2 are

$$
\begin{gathered}
x=x_{0}+\Delta x \\
x^{\prime}=x_{0}^{\prime}+\Delta x^{\prime}
\end{gathered}
$$




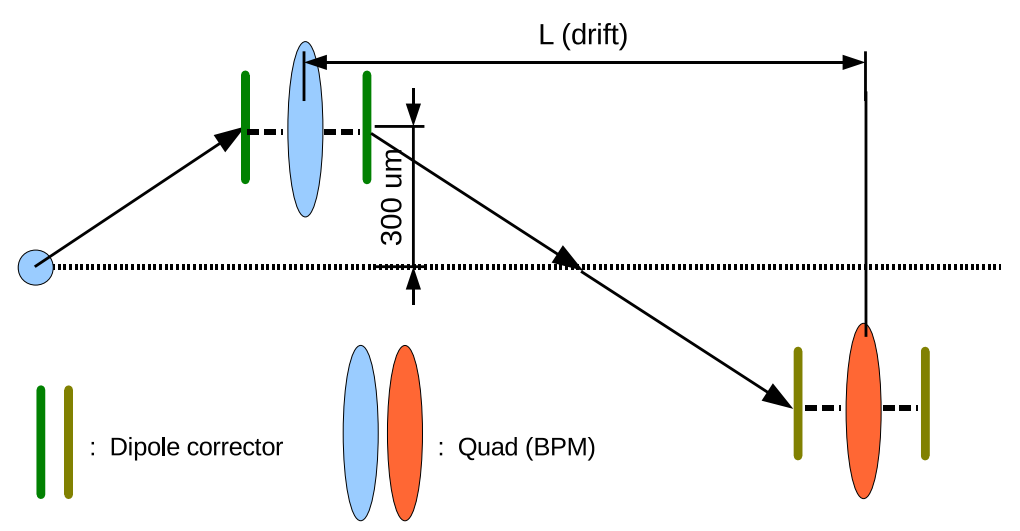

Figure 4: Model for the analytical treatment of one to one correction.

$$
\begin{gathered}
y=y_{0}+\Delta y \\
y^{\prime}=y_{0}^{\prime}+\Delta y^{\prime}
\end{gathered}
$$

Insert formulae (4)-(9) into formula (3), and by using $\left.\left.\sigma_{y}{ }^{2}=<y^{2}>,{\sigma_{y}^{\prime}}^{2}=<y^{\prime 2}\right\rangle, \sigma_{y y^{\prime}}=<y y^{\prime}\right\rangle$, the normalized emittance can be written as [5]

$$
\left(\gamma \epsilon_{y}\right)^{2}=\gamma^{2}\left[\left(\sigma_{y}{ }^{2}+<\Delta y^{2}>\right) \cdot\left(\sigma_{y^{\prime}}^{2}-<\Delta y^{\prime 2}>\right)-\left(\sigma_{y y^{\prime}}^{2}-<\Delta y \Delta y^{\prime}>\right)^{2}\right]
$$

Expand the right side of formula (10) and by using

$$
\begin{gathered}
\left(\gamma \epsilon_{y}\right)^{2}=\gamma^{2}\left(\sigma_{y}{ }^{2} \cdot \sigma_{y^{\prime}}^{2}-\sigma_{y y^{\prime}}^{2}\right) \\
\sigma_{y}^{2}=\epsilon_{y} \beta_{y} \\
{\sigma_{y}^{\prime}}^{2}=\frac{\epsilon_{y}}{\beta_{y}} \\
\sigma_{y y^{\prime}}=-2 \epsilon_{y} \alpha_{y}
\end{gathered}
$$

the normalized emittance can be written as

$$
\gamma \epsilon_{y}=\gamma \epsilon_{y 0} \sqrt{1+2 \Delta \gamma \epsilon / \gamma \epsilon_{y 0}}
$$

where

$$
\Delta \gamma \epsilon=\frac{\gamma}{2}\left(\frac{1+\alpha^{2}}{\beta} \Delta y^{2}+2 \alpha \Delta y \Delta y^{\prime}+\beta \Delta y^{\prime 2}\right)
$$

We use the model as shown in Figure 4, initial longitudinal starting point is the middle of the drift in a FODO cell. The beam centroid is on the survey axis (treated as straight line here) at first. At the starting point, the beam 
gets a dipole kick which makes the beam center to travel through the next quadrupole center. The normalized dipole corrector strength is

$$
K_{0}=2 \sigma_{\text {quad }} / L_{\text {drift }}
$$

where $L_{\text {drift }}$ denotes the length of the drift between two quads, $\sigma_{\text {quad }}$ the RMS value of quadrupole offsets.

After passing by this dipole corrector, particle 1 has a change in the vertical divergence of $\Delta y_{0}^{\prime}=K_{0}$. For particle 2 , the change is $\Delta y_{1}^{\prime}=K_{0} /\left(1+\delta_{p}\right)$. The kick of the next dipole corrector is divided into two parts, with the quadrupole being sandwiched between them. The first part cancels the kick from the previous dipole corrector $K_{0}$, and the second part will kick the beam to the next quadrupole center. Assuming thin lens approximation, at the location of the first quadrupole, the difference of coordinate $y$ between particle 1 and 2 can be expanded into Taylor series

$$
\Delta y=\frac{k_{0} L}{1+\delta_{p}}-k_{0} L=k_{0} L\left(-\delta_{p}+\delta_{p}^{2}-\delta_{p}^{3}+\ldots\right)
$$

We will observe the beam emittance at the exit of the first quadrupole, where the difference of coordinate $y$ between particle 1 and 2 is presented in formula (18), and the difference of divergence is

$$
\Delta y^{\prime}=K_{1} \Delta y
$$

Insert formulae (18) and (19) into formulae (15) and (16), we can get the new emittance after dispersive and chromatic growth in this small unit. With one unit containing two parts of two dipole correctors and the next quadrupole, we can repeat this process along the linac and calculate the emittance. One thing to note is that: at the observatory point which is the exit of the quadrupole, $\alpha_{y}$ always has opposite sign with the normalized quadrupole strength $K_{1}$. That means for vertical emittance, the cross term of $\Delta y$ and $\Delta y^{\prime}$ in formula (16) always cancels the contribution of the other two terms.

With acceleration, the RMS energy spread will decrease as $\sigma_{p} E_{0} / E$, and this parameter should be updated accordingly in calculating $\Delta y$ and $\Delta y^{\prime}$.

We can also observe the beam emittance at the center of each quadrupole. For this case, one unit is from the center of one quadrupole to the center of next quadrupole. The difference of vertical coordinate $\Delta y$ between particle 1 and 2 will be the same.

$$
\Delta y=\frac{k_{0} L}{1+\delta_{p}}-k_{0} L=k_{0} L\left(-\delta_{p}+\delta_{p}^{2}-\delta_{p}^{3}+\ldots\right)
$$

The difference of vertical divergence $\Delta y^{\prime}$ between particle 1 and 2 should be a half of the previous one.

$$
\Delta y^{\prime}=K_{1} \Delta y / 2
$$

At the center of the quadrupole, we can treat $\alpha=0$ and rewrite formula (16) as

$$
\Delta \gamma \epsilon=\frac{\gamma}{2}\left(\frac{1}{\beta} \Delta y^{2}+\beta \Delta y^{\prime 2}\right)=\frac{\gamma}{2}\left(\frac{1}{\beta}+\frac{\beta K_{1}^{2}}{4}\right) \Delta y^{2}
$$




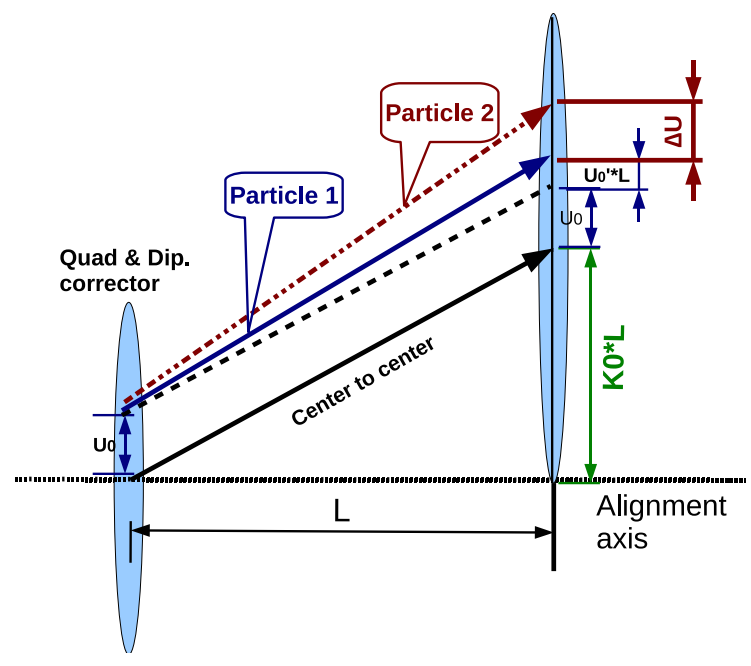

Figure 5: Sketch of the two-particle model.

\subsection{Method Two}

The square of the 2-D projected physical emittance can be calculated as the determinant of the following matrix

$$
\epsilon^{2}=\left(\begin{array}{cc}
\sigma_{y_{0}}^{2}+\sum<\Delta y_{i}^{2}> & 0 \\
0 & \sigma_{y_{0}^{\prime}}^{2}+\sum<\Delta y_{i}^{\prime 2}>
\end{array}\right)
$$

where $\left\langle\Delta y_{i}{ }^{2}>\right.$ denotes the average over all the particles at the $i^{\text {th }}$ error source, $\sum$ the sum over all the error sources, $\Delta y$ the relative position change with respect to the bunch centroid, and $\Delta y^{\prime}$ the relative angle change with respect to the bunch centroid.

In the case of one to one correction scheme, in principle only the dispersion generated by the dipole corrector (dispersive effect) and the quadrupole kick at the dispersive region (chromatic effects) contribute to the change of position and angle. So basically the dispersive effect from the dipole corrector introduces a wider spread in $\Delta y$ at the quadrupole locations, and the chromatic effects of the quadrupole introduces a wider spread in $\Delta y^{\prime}$ afterwards. These two effects happen at different locations and can be treated separately, as shown in Figure 5, which is a similar two-particle model as used in menthod one.

As illustrated in Figure 6, the dispersive emittance growth is a local effect, which is also the reason that why it can be corrected "locally". Four vertical dipole correctors are used to make one closed bump, at the location of either focusing or defocusing quadrupole. The emittance growth generated in this bump stays the same as the bunch travels through the following cells which has no dispersion. The effect that there is a larger vertical emittance growth at the defocusing quadrupole with the same bump amplitude, will be explained later in this section.

The "local" contribution to emittance growth from either position change or angle change can be propagated to the cells downstream, by using the transport matrix listed below.

The 2-D transport matrix between location 1 and 2 is

$$
\mathbf{M}_{\mathbf{1}, \mathbf{2}}=\left(\begin{array}{ll}
R_{11} & R_{12} \\
R_{21} & R_{22}
\end{array}\right)
$$

where 

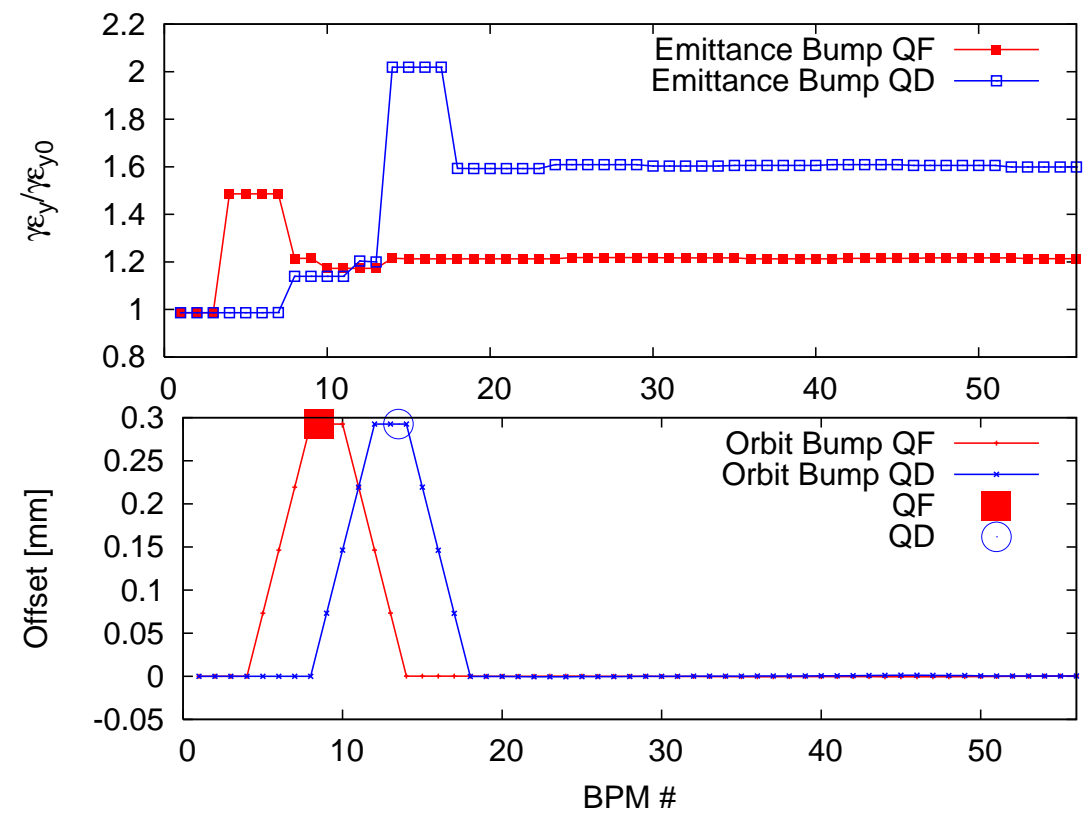

Figure 6: Demonstration of the "local" property of the dispersive emittance growth, by making a closed vertical bump and observe the vertical emittance by simulation. Top: relative vertical emittance; bottom: beam orbit and quadrupole location.

$$
\begin{gathered}
R_{11}=\sqrt{\frac{\beta_{2}}{\beta_{1}}}\left(\cos \Delta \phi+\alpha_{1} \sin \Delta \phi\right) \\
R_{12}=\sqrt{\beta_{2} \beta_{1}} \sin \Delta \phi \\
R_{21}=-\frac{1+\alpha_{1} \alpha_{2}}{\sqrt{\beta_{2} \beta_{1}}} \sin \Delta \phi+\frac{\alpha_{1}-\alpha_{2}}{\sqrt{\beta_{2} \beta_{1}}} \cos \Delta \phi \\
R_{22}=\sqrt{\frac{\beta_{1}}{\beta_{2}}}\left(\cos \Delta \phi-\alpha_{2} \sin \Delta \phi\right)
\end{gathered}
$$

Before we start the analytical derivation, let us look at the example with two FODO cells, as shown in Figure 7. There are eight vertical dipole correctors, which build up two closed vertical bumps for cell 1 and cell 2 . The height of the bump equals the RMS quadrupole offset $\sigma_{\text {quad }}=300 \mu \mathrm{m}$. As shown in Figure 7 (bottom), the first dipole corrector is next to the first quadrupole, which dipole kick is totally cancelled by the second dipole corrector. The third dipole corrector is right after the second quadrupole and it kicks the beam back to the alignment axis. The fourth dipole corrector cancels the kick from the third one. If the strength of the second and fourth quadrupole is set to zero, it is obvious to see that the change of all the particles' position and angle is zero at the exit of the bump. That means there is no emittance growth, as shown by the blue dotted curve in Figure 7 (top). On the other hand, one can also conclude that over a long linac, the main contribution on the dispersive emittance growth is from the quadrupole kick only at the dispersion region, as shown by the red and magenta curves in Figure 7 (top).

Note that for this method, we will use a model such that the focusing (or defocusing) quadrupole is perfectly aligned, and only the defocusing (or focusing) quadrupole has an offset which RMS value is $\sigma_{\text {quad }}$. For a linac with $n$ FODO cells, equivalently there will be $2 n$ FODO cells in the model being used as only every other quadrupole has offset. 


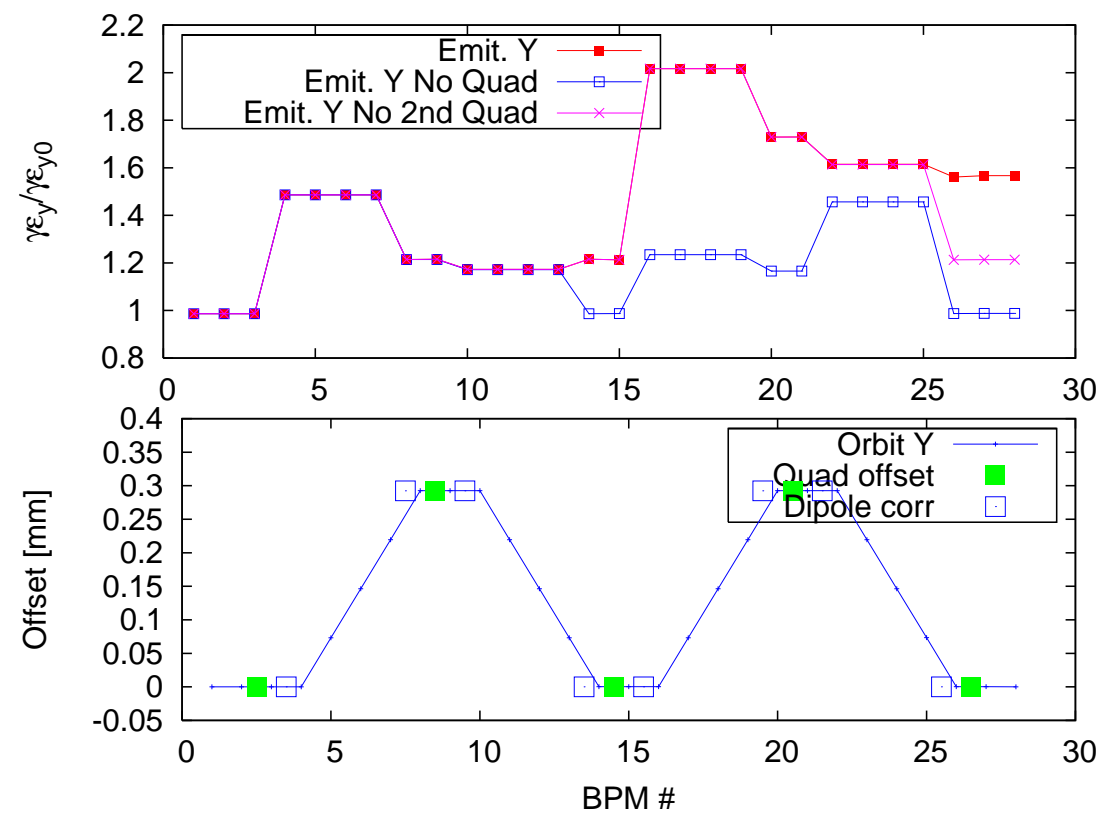

Figure 7: Optics model being used for method two. Top: relative vertical emittance for three different cases; bottom: beam orbit and quadrupole (dipole corrector) location.

\subsubsection{No acceleration}

For the case without acceleration, one can use the physical coordinates $\left(y, y^{\prime}\right)$, and treat the terms $\Delta y$ and $\Delta y^{\prime}$ separately in formula (23). As the bunch travels through the linac, the change in angle spread $\Delta y^{\prime}$ will propagate, and at the center of any quadrupole downstream we have

$$
\begin{aligned}
& <\Delta y_{i}{ }^{2}>=\sum_{j=1}^{i-1} R_{12_{j, i}}<\Delta y_{j}^{\prime 2}> \\
& <\Delta y_{i}^{\prime 2}>=\sum_{j=1}^{i-1} R_{22_{j, i}}<\Delta y_{j}^{\prime 2}>
\end{aligned}
$$

Insert $R_{12}$ and $R_{22}$ into the above equations, and applying

$$
\begin{gathered}
\beta_{1, j}=\beta_{2, j}=\beta \\
\alpha_{1, j}=\alpha_{2, j}=0 \\
\sum_{j=1}^{i-1} \cos ^{2} \Delta \phi=\sum_{j=1}^{i-1}\left(\frac{1}{2}+\frac{1}{2} \cos 2 \Delta \phi\right) \\
\sum_{j=1}^{i-1} \sin ^{2} \Delta \phi=\sum_{j=1}^{i-1}\left(\frac{1}{2}+\frac{1}{2} \sin 2 \Delta \phi\right) \\
\sum_{j=1}^{i-1}\left(\frac{1}{2} \cos 2 \Delta \phi\right)=0
\end{gathered}
$$




$$
\sum_{j=1}^{i-1}\left(\frac{1}{2} \sin 2 \Delta \phi\right)=0
$$

We have

$$
\begin{aligned}
& <\Delta y_{i}^{2}>=\sum_{j=1}^{i-1}\left(\frac{1}{2}\right) \beta^{2}<\Delta y_{j}^{\prime 2}> \\
& <\Delta y_{i}^{\prime 2}>=\sum_{j=1}^{i-1}\left(\frac{1}{2}\right)<\Delta{y_{j}^{\prime}}^{2}>
\end{aligned}
$$

At each quadrupole, we have

$$
<\Delta y_{j}^{\prime 2}>=<\left(K_{1} \cdot \sigma_{Q} \cdot \delta_{p}\right)^{2}>=\left(K_{1} \cdot \sigma_{Q}\right)^{2}<\left(\delta_{p}\right)^{2}>=\left(K_{1} \cdot \sigma_{Q} \cdot \sigma_{p 0}\right)^{2}
$$

Insert formulae (37)-(39) into formula (23), the physical vertical emittance at the $n^{\text {th }}$ cell is

$$
\epsilon_{n}=\sqrt{\left({\sigma_{y_{0}}}^{2}+0.5 \cdot n \cdot \beta^{2} \cdot\left(K_{1} \cdot \sigma_{Q} \cdot \sigma_{p 0}\right)^{2}\right)\left({\sigma_{y_{0}^{\prime}}}^{2}+0.5 \cdot n \cdot\left(K_{1} \cdot \sigma_{Q} \cdot \sigma_{p 0}\right)^{2}\right)}
$$

From formula (40), one can conclude that there is more vertical emittance growth from the defocusing quadrupole, given the same dispersion. That explains the observation in Figure 6. To evaluate the emittance growth properly, one can use the average beta function $\beta_{y}=\sqrt{\beta_{y, Q D} \cdot \beta_{y, Q F}}$ instead.

\subsubsection{With acceleration}

For the case with acceleration, the beam energy is increased along the linac and the RMS energy offset is decreased accordingly.

$$
\sigma_{p}=\sigma_{p 0} \frac{E_{0}}{E_{n}}
$$

where $E_{0}$ denotes the initial beam energy and $E_{n}$ the energy at the $n^{\text {th }}$ cell.

At the same time, the transverse coordinates also shrink under adiabatic damping.

$$
\begin{aligned}
& y=y_{0} \sqrt{\frac{E_{0}}{E_{n}}} \\
& y^{\prime}=y_{0}^{\prime} \sqrt{\frac{E_{0}}{E_{n}}}
\end{aligned}
$$

Formula (39) can be rewritten as

$$
<\Delta y_{j}^{\prime 2}>=\left(\sqrt{\frac{E_{n}}{E_{0}}} K_{1} \cdot \sigma_{Q} \cdot \sigma_{p 0} \frac{E_{0}}{E_{n}}\right)^{2}=\left(K_{1} \cdot \sigma_{Q} \cdot \sigma_{p 0}\right)^{2} \cdot \frac{E_{0}}{E_{n}}
$$

Insert formulae (37), (38) and (44) into formula (23), the normalized emittance at the $n^{\text {th }}$ cell can be calculated by integration over cell numbers, assuming that the energy gain is the same in each cell.

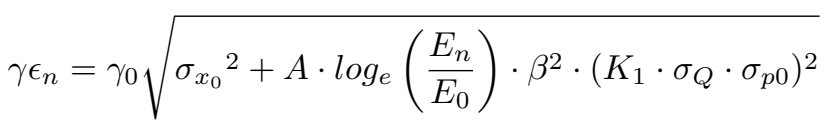

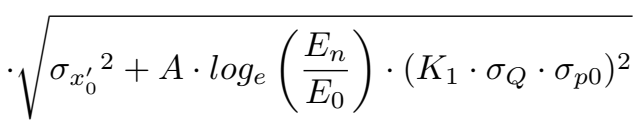

where $A$ denotes a contant which is dependent on the initial energy and energy gain per cell. 


\subsection{Comparison and discussion}

We apply method one (and two) to ILC main linac which has 300 quadrupoles in all (distributed in 150 FODO cells). The parameters being used are listed in Table 1. For method two, formula (45) is rewritten and the normalized emittance at the $n^{\text {th }}$ cell is

$$
\begin{aligned}
& \gamma \epsilon_{n}=\gamma_{0} \sqrt{\sigma_{x_{0}}{ }^{2}+0.5 \int_{0}^{n} \frac{15}{15+0.8 n} d n \cdot \beta^{2} \cdot\left(K_{1} \cdot \sigma_{Q} \cdot \sigma_{p 0}\right)^{2}} \\
& \cdot \sqrt{{\sigma_{x_{0}^{\prime}}}^{2}+0.5 \int_{0}^{n} \frac{15}{15+0.8 n} d n \cdot\left(K_{1} \cdot \sigma_{Q} \cdot \sigma_{p 0}\right)^{2}} \\
& =\gamma_{0} \sqrt{{\sigma_{x_{0}}}^{2}+0.5 \cdot 18.75 \cdot\left(\log _{e}(15+0.8 n)-\log _{e}(15)\right) \cdot \beta^{2} \cdot\left(K_{1} \cdot \sigma_{Q} \cdot \sigma_{p 0}\right)^{2}}
\end{aligned}
$$

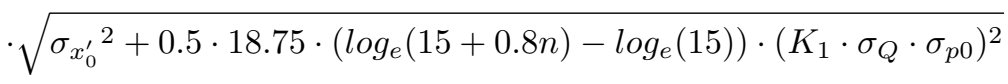

Without and with acceleration, the comparison between analytical results of method one (and two) and the simulation is shown in Figure 8 and Figure 9 respectively. For the case without acceleration, the agreement between simulation and method two is very well, where the average beta function $\beta_{y}=\sqrt{\beta_{y, Q D} \cdot \beta_{y, Q F}}=62.5 m$ is used. For method one there is a small difference if compared with the simulation result.

TABLE 1 Relevant parameters

\begin{tabular}{r|l}
\hline parameter & value \\
\hline \# of quad & 300 \\
Drift length $[\mathrm{m}]$ & 39 \\
Quad offset $\sigma_{\text {quad }}[\mu \mathrm{m}]$ & 300 \\
Norm. quad strength $\left[\mathrm{m}^{-1}\right]$ & \pm 0.03 \\
$\alpha_{y}$ at exit of quad & $1.9(-0.6)$ \\
$\beta_{y}$ at quad $[\mathrm{m}]$ & $30(130)$ \\
Average $\beta_{y}$ at quad $[\mathrm{m}]$ & 62.5 \\
Energy gain in each cell $[\mathrm{GeV}]$ & 1.6 \\
Initial energy spread $\sigma_{p}$ & 0.015 \\
Initial bunch length $\sigma_{z}[\mu \mathrm{m}]$ & 300 \\
Initial emittance $\gamma \epsilon_{y 0}[\mathrm{~nm}]$ & 40 \\
RF frequency $[\mathrm{GHz}]$ & 1.3 \\
RF phase $[$ degree $]$ & -4.6 \\
\hline
\end{tabular}

With acceleration, there is a big difference between the simulation and analytical results. In the analytical treatment, the RMS energy spread decreases as $\sigma_{p} E_{0} / E$, which means there is a smaller dispersive emittance growth as the energy increases. In the simulation, however, we observe a large emittance growth, even near the end of the linac. It is the finite bunch length $\sigma_{z}=300 \mu m$ which causes this effect. The bunch length associated with the proper beam-RF phase can provide BNS damping to mitigate the wake field effects.

As discussed before, in a linear accelerator there is almost no longitudinal oscillation in a bunch, which means particle's longitudinal position with respect to the bunch center is effectively "frozen". When a bunch pass by a RF cavity, the contribution to the RMS energy spread is

$$
\Delta \sigma_{p}=\frac{V_{r f} \cdot\left(\cos \left(\phi_{0}+\omega_{r f} \cdot \sigma_{z}\right)-\cos \left(\phi_{0}\right)\right)}{E}
$$

where $V_{r f}$ denotes the RF accelerating voltage in $G e V, \phi_{0}$ the beam-RF phase, $\omega_{r f}$ the RF angular frequency, $E$ the beam energy at this RF cavity location. 

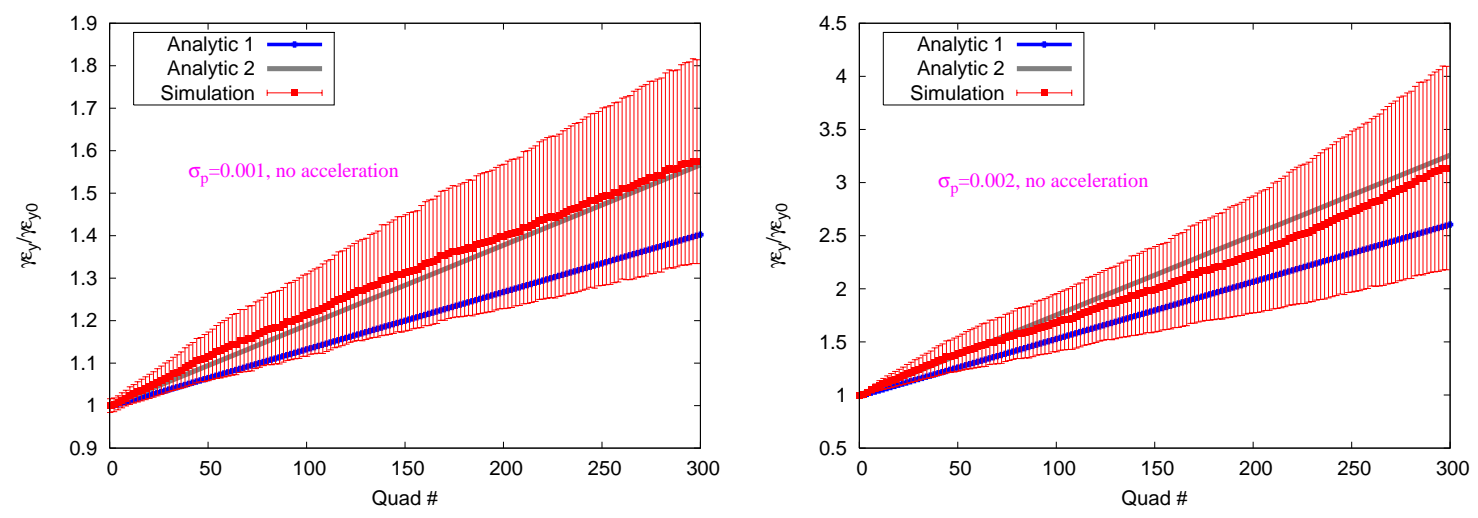

Figure 8: Comparison of relative normalized emittance, between analytical results and simulation, without acceleration. Left: $\delta_{p}=0.001 ;$ Right: $\delta_{p}=0.002$.
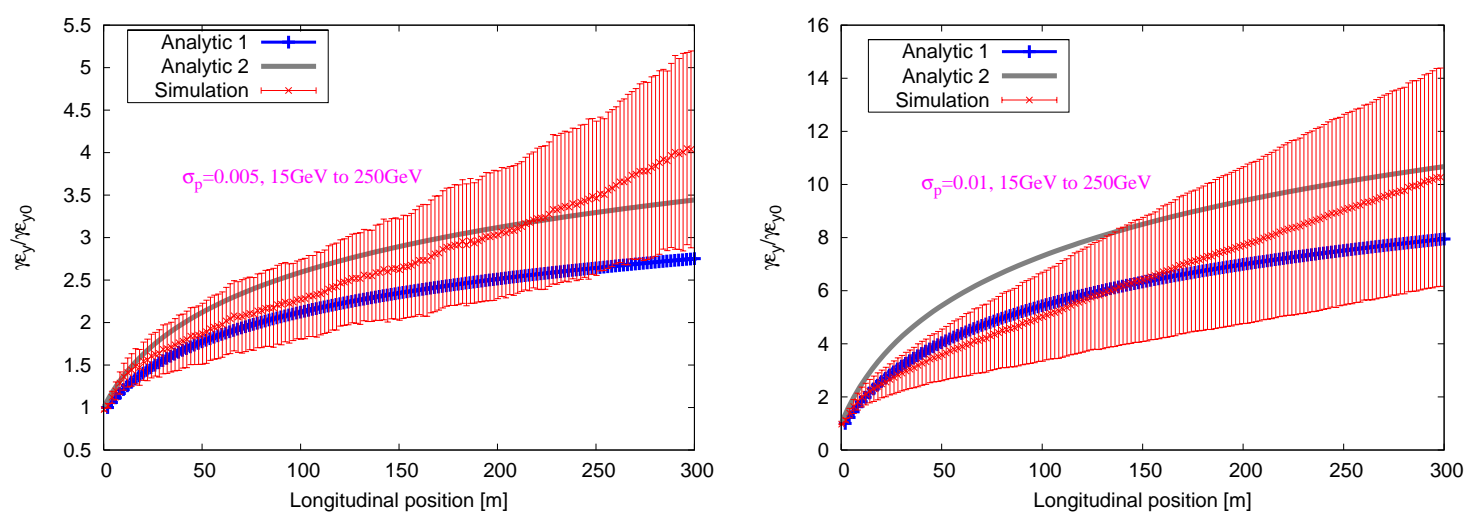

Figure 9: Comparison of relative normalized emittance, between analytical results and simulation, with acceleration. Left: $\delta_{p}=0.005 ;$ Right: $\delta_{p}=0.01$.

At the end of the linac, the increasement of the RMS energy spread is the sum over all the RF acceleration locations.

$$
\Delta_{e n d} \sigma_{p}=\sum \frac{V_{r f} \cdot\left(\cos \left(\phi_{0}+\omega_{r f} \cdot \sigma_{z}\right)-\cos \left(\phi_{0}\right)\right)}{E}
$$

An example of this effect is shown in Figure 10, where the evolvement of RMS energy spread is plotted along the linac. The blue curve represents the contribution from formula (48). In the zoom-in figure, it is clearly shown that at the second part of the linac, the contribution from formula (48) is so large that it will increase the RMS energy spread by almost a half. For the case with zero bunch length, the RMS energy spread gotten from simulation is plotted with magenta dots, while the results from formula $\sigma_{p}=\sigma_{p 0} E_{0} / E$ is plotted with magenta curve.

As there is no finite bunch length effect in the model of both method one and two, we set the bunch length to be zero and redo the simulation. The new comparison is shown in Figure 11 (left), where the shape of the simulated emittance growth is similar to the analytical result now, although there is still differences in the amplitude. We can fit formula (45) to the simulation results, by varying the parameters in formula (45), as shown in Figure 11 (right).

Using another two initial RMS energy offset $\left(\delta_{p}=0.0075\right.$ and $\left.\delta_{p}=0.01\right)$, the simulated emittance growth is again compared with the analytical estimations from formula (45), by applying the same fitting parameter from last case of $\delta_{p}=0.005$. A pretty good agreement is observed, as shown in Figure (12). 


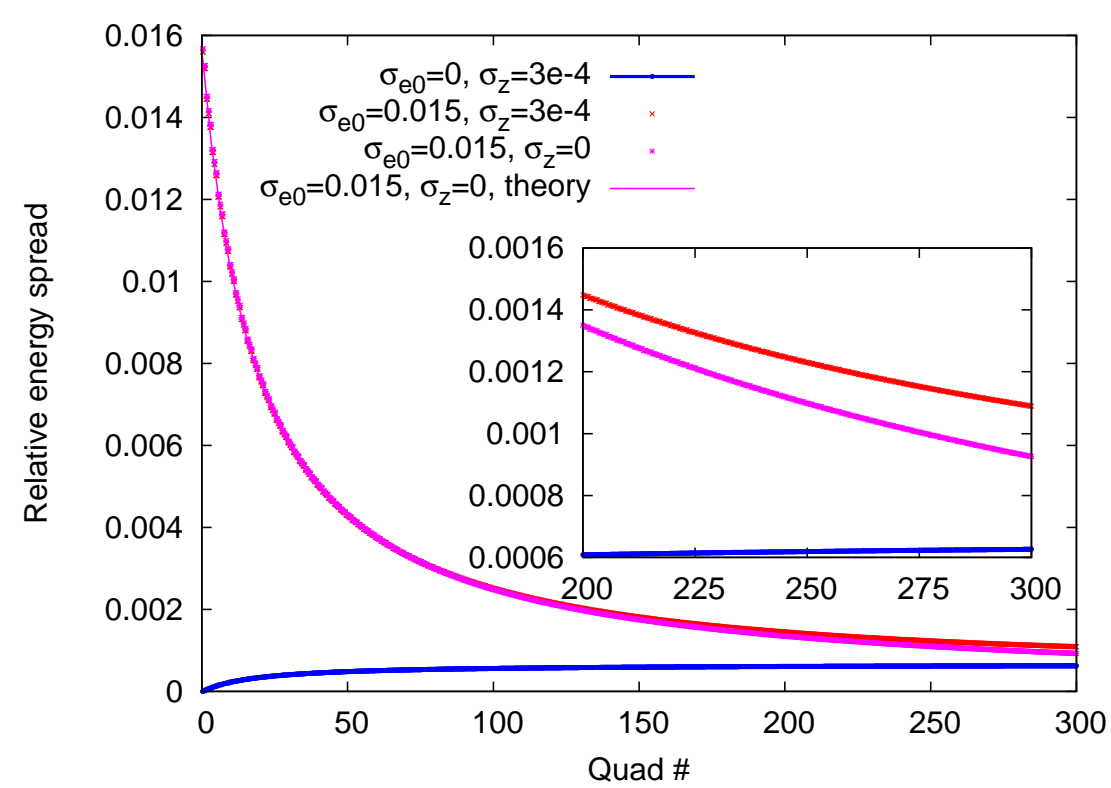

Figure 10: Evolvement of RMS energy spread along the linac. The red curve represents the energy spread from initial energy spread plus contribution of finite bunch length.
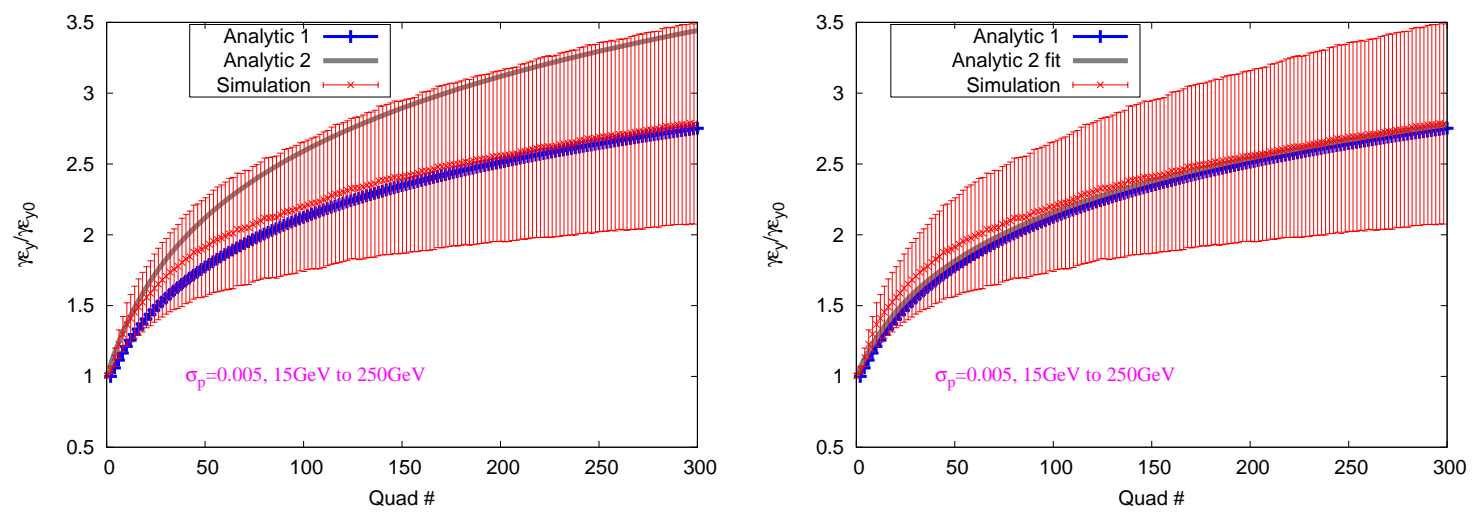

Figure 11: Comparison of relative normalized emittance, between analytical results and simulation, with acceleration. Left: $\delta_{p}=0.005$; Right: $\delta_{p}=0.005$, fit parameters in formula (45) (method two).
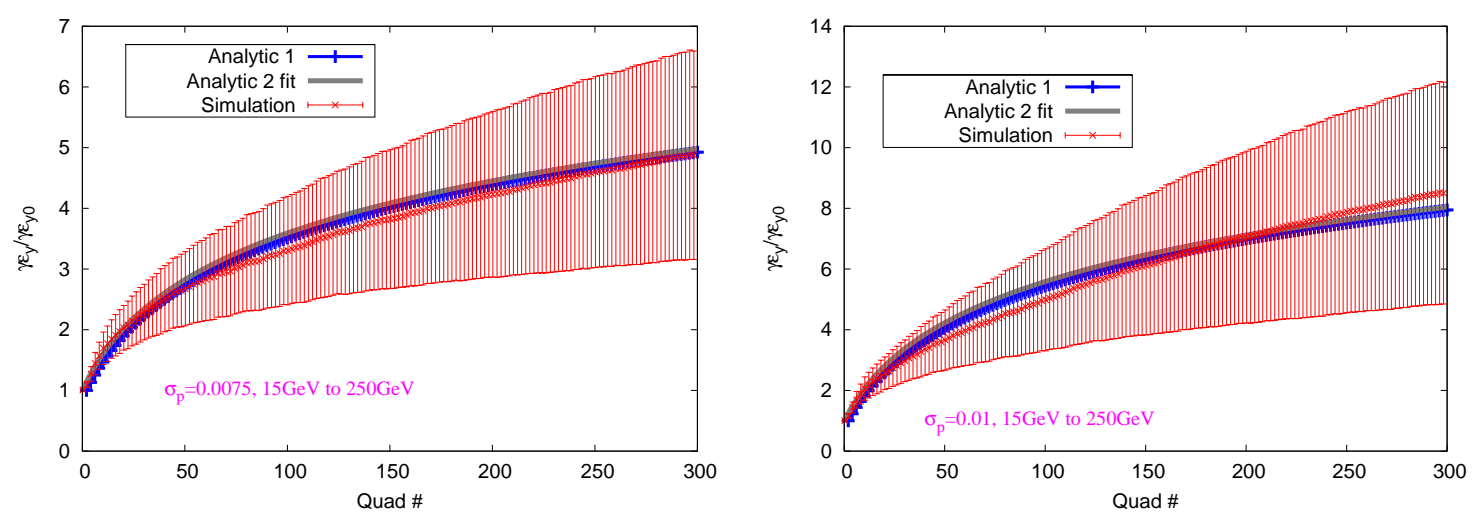

Figure 12: Comparison of relative normalized emittance, between analytical results and simulation, with acceleration. Left: $\delta_{p}=0.0075$; Right: $\delta_{p}=0.01$. Use the same fitting parameters calculated in the case of $\delta_{p}=0.005$. 


\section{Acknowledgement}

The authors would like to thank Dr. A. Chao for helpful discussions.

This work was supported by the DOE under Contract DE-AC02-76SF00515.

\section{References}

[1] C. Adolphsen et al., PAC 93, page 417-419.

[2] T.O. Raubenheimer and R. Ruth, Nucl. Instr. and Meth. A302, 191 (1991); T.O. Raubenheimer, Nucl. Instr. and Meth. A306, 61 (1991).

[3] T.O. Raubenheimer and D. Schulte, Proc. Part. Accel. Conf. (PAC99), New York (1999) and CERN-PS 99-024-LP (1999).

[4] K. Kubo, Lecture on 1st ILC school, Japan (2006).

[5] T.O. Raubenheimer, Phys. Rev. ST Accel. Beams 3, 121002 (2000).

[6] T.O. Raubenheimer, PhD thesis, Stanford U., SLAC-R-387 (1991).

\section{: Appendix ADefinition of dispersion and emittance}

From the statistical point of view, the 2-D projected emittance can be defined as

$$
\epsilon=\sqrt{\left(<x^{2}>-<x>^{2}\right)\left(<x^{\prime 2}>-<x^{\prime}>^{2}\right)-\left(<x x^{\prime}>-<x><x^{\prime}>\right)^{2}}
$$

where the bracket means an average over all the macro-particles.

At the same time, the linear dispersion corrected emittance can be defined as

$$
\begin{array}{r}
\epsilon=\left[\left(<\left(x-D_{x} \delta\right)^{2}>-<x-D_{x} \delta>^{2}\right)\left(<\left(x^{\prime}-D_{x}^{\prime} \delta\right)^{2}>-<x^{\prime}-D_{x}^{\prime} \delta>^{2}\right)\right. \\
\left.-\left(<\left(x-D_{x} \delta\right)\left(x^{\prime}-D_{x}^{\prime} \delta\right)>-<x-D_{x} \delta><x^{\prime}-D_{x}^{\prime} \delta>\right)^{2}\right]^{0.5}
\end{array}
$$

The "dispersion" ( and the derivative of the "dispersion" ) of the beam can be calculated by

$$
\begin{gathered}
D_{x}=(<x \delta>-<x><\delta>) /\left(<\delta^{2}>-<\delta>^{2}\right) \\
D_{x}^{\prime}=\left(<x^{\prime} \delta>-<x^{\prime}><\delta>\right) /\left(<\delta^{2}>-<\delta>^{2}\right)
\end{gathered}
$$

\section{: Appendix BBenchmark of the code}

\section{Optics}

The parameters of one FODO cell and the beam used in the simulation is listed in Table 1. The beta function which is calculated statistically from the simulation agrees well with the MADX TWISS calculation. 

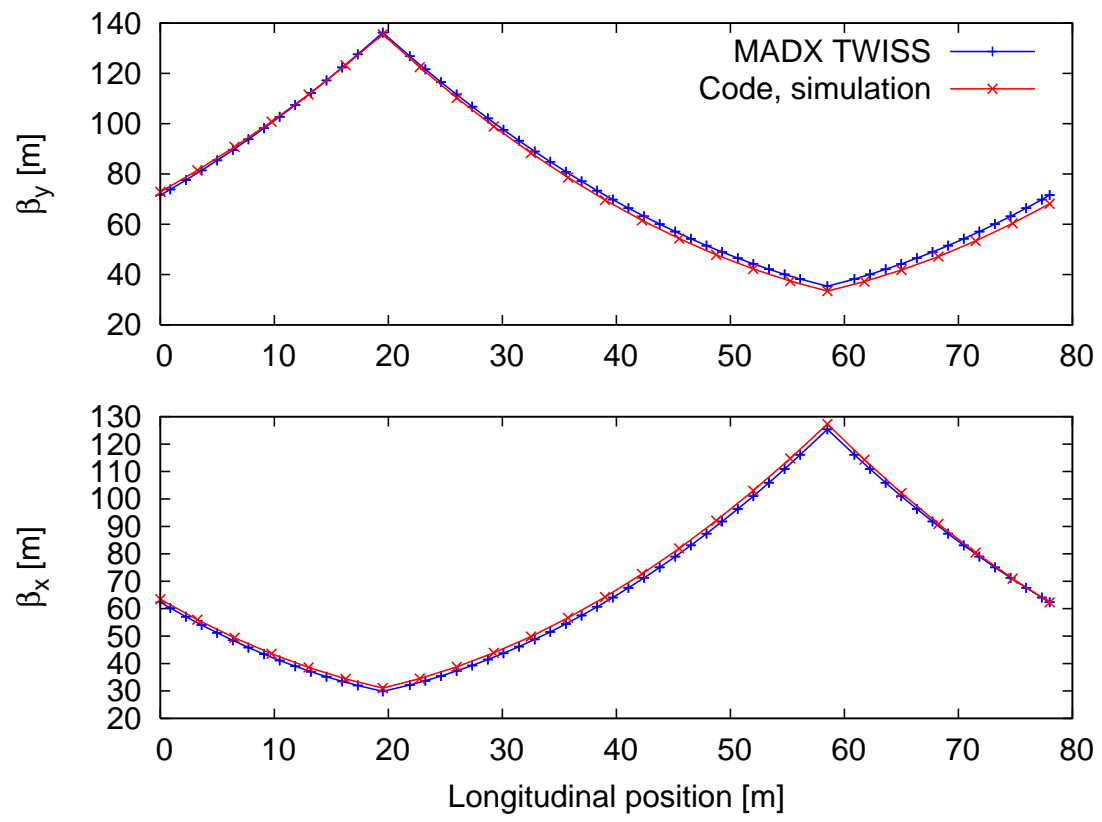

Figure 13: Benchmark of the beta function in one FODO cell, between MADX TWISS output and the simulation results of this code.

TABLE 1 Relevant parameters.

\begin{tabular}{r|l}
\hline parameter & value \\
\hline \# of quad & 2 \\
Drift length $[\mathrm{m}]$ & 39 \\
Cell length $[\mathrm{m}]$ & 78 \\
Norm. quad strength $\left[\mathrm{m}^{-1}\right]$ & \pm 0.03 \\
$\beta_{y}$ at exit of quad & $30(130)$ \\
Initial energy spread $\sigma_{p}$ & 0.015 \\
Initial emittance $\gamma \epsilon_{y 0}[\mathrm{~nm}]$ & 40 \\
Initial emittance $\gamma \epsilon_{x 0}[\mu \mathrm{m}]$ & 10 \\
\hline
\end{tabular}

\section{Orbit}

The orbit of the bunch centroid (represented by a single particle with initial coordinates $(0,0,0,0,0,0)$ is compared with the orbit of the bunch (10,000 macro-particles) along the ILC main linac which is roughly $11 \mathrm{~km}$ long. The quadrupole has an offset of $\sigma_{\text {quad }}=300 \mu \mathrm{m}$ in both horizontal and vertical plane. Good agreement is found in the comparison.

\section{Filamentation (chromatic and dispersive)}



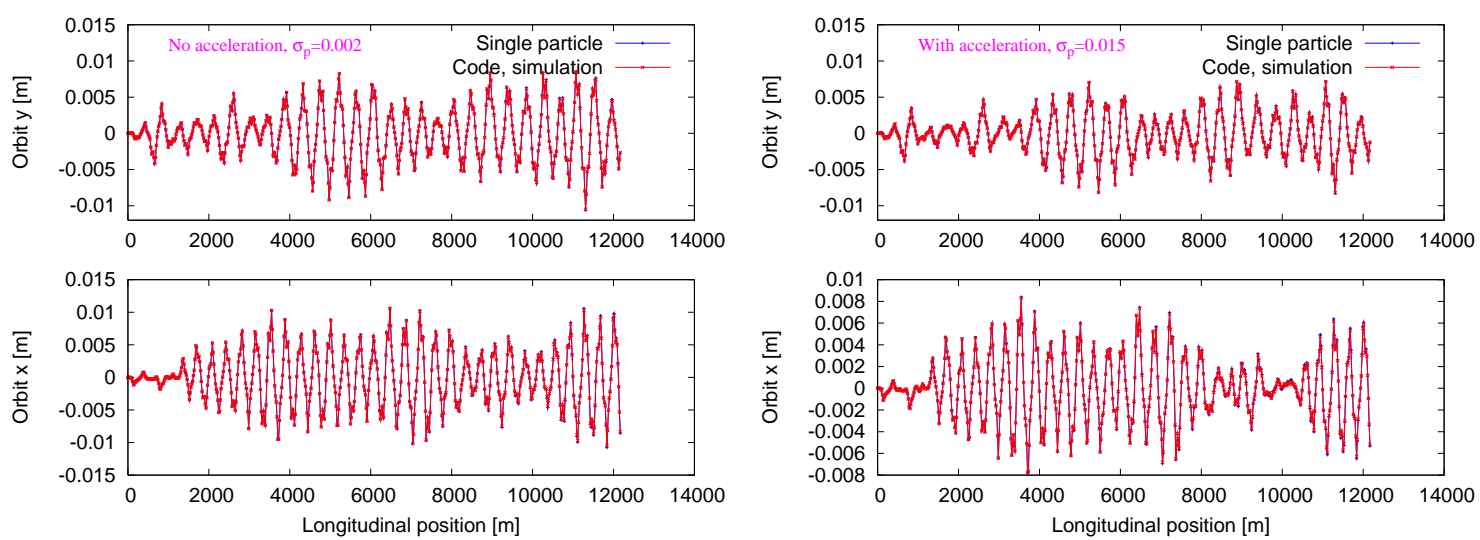

Figure 14: Comparison between the single particle orbit (initial coordinates $(0,0,0,0,0,0))$ and the orbit of a bunch (initial bunch centroid at $(0,0,0,0,0,0))$ along ILC main linac, with quadrupole random offset $\sigma_{\text {quad }}=300 \mu m$. Left: without acceleration, with a RMS energy spread of $\delta_{p}=0.002$; Right: with acceleration, with a RMS energy spread of $\delta_{p}=0.015$.
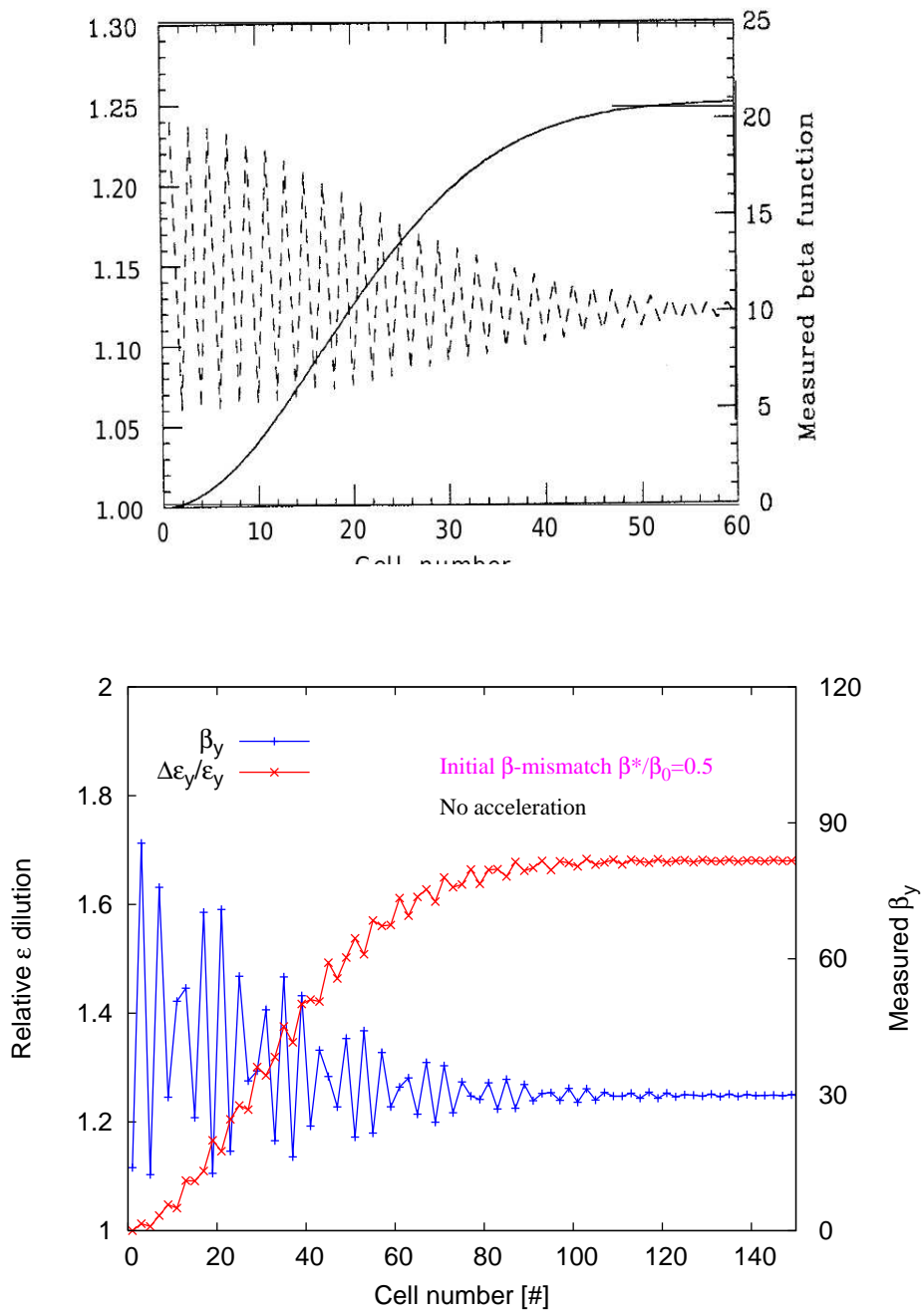

Figure 15: Benchmark of chromatic filamentation between the results of this code and ref [6], initial beta mismatch is $\beta^{*} / \beta^{0}=0.5$. Top: T. Raubenheimer's result, $\delta_{p}=0.01$; Bottom: result of this code, $\delta_{p}=0.015$. 

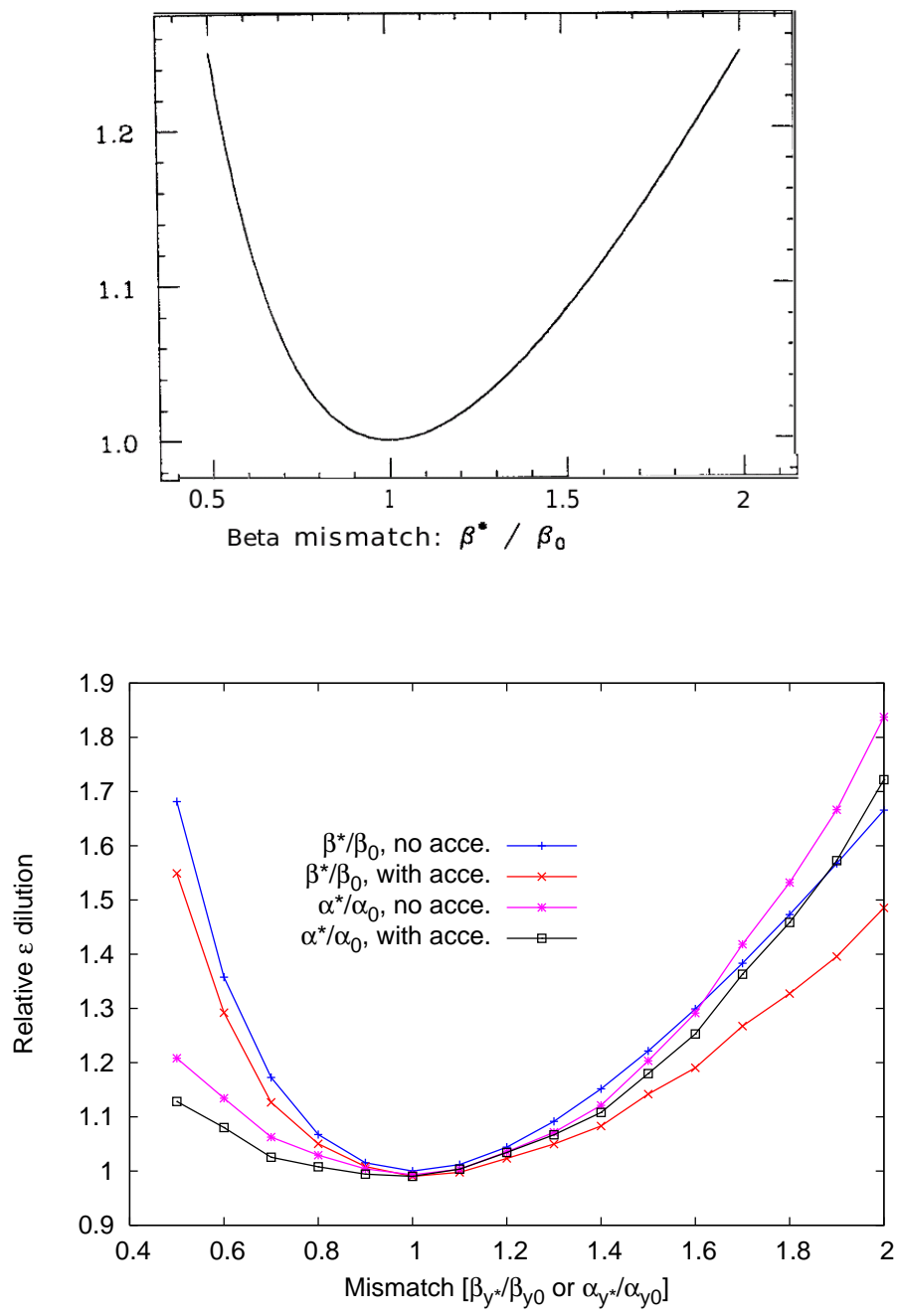

Figure 16: Benchmark of chromatic filamentation between the results of this code and ref [6], initial beta mismatch is varied from $\beta^{*} / \beta^{0}=0.5$ to $\beta^{*} / \beta^{0}=2$. Top: T. Raubenheimer's result, $\delta_{p}=0.01$; Bottom: result of this code, $\delta_{p}=0.015$. 

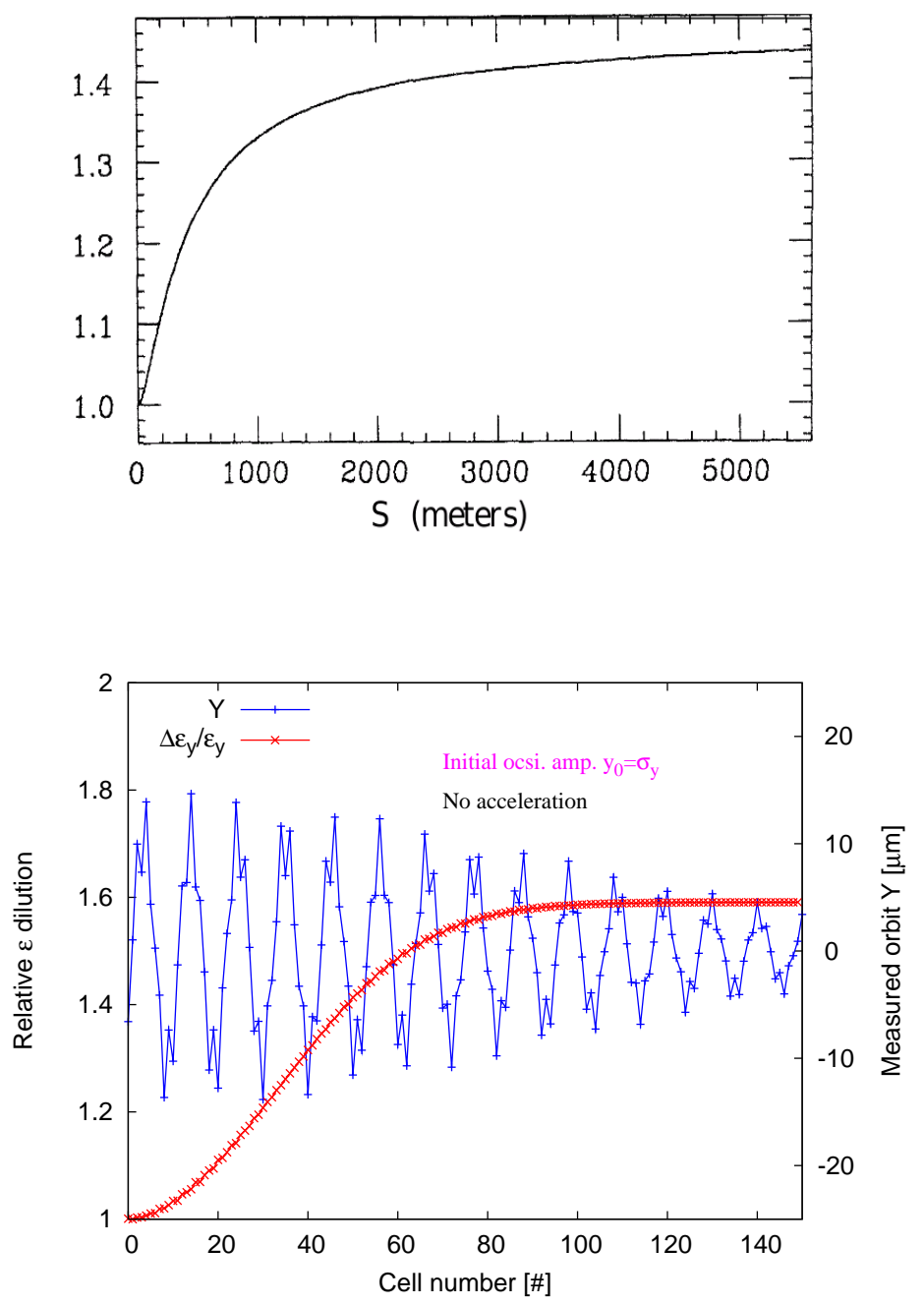

Figure 17: Benchmark of dispersive filamentation between the results of this code and ref [6], initial beam offset is $\Delta_{y}=1 \sigma_{y}$. Top: T. Raubenheimer's result, $\delta_{p}=0.01$; Bottom: result of this code, $\delta_{p}=0.015$. 